\title{
Hypertonie
}

\section{Beeindruckende Effekte einer gesunden Ernährung}

\section{Einmal mehr zeigt eine Studie, wie wirkungsvoll Allgemeinmaßnahmen einen Bluthochdruck verbessern können.}

_ In einer beim US-Herzkongress vorgestellten Studie aus dem Beth Israel Deaconess Medical Center in Boston hatten sich 412 Hypertoniker vier Wochen lang entweder salzarm und/oder entsprechend US-Hochdruckleitlinien besonders „Blutdruck-gesund“ ernährt (DASH-Diät: Obst, Gemüse, Vollkorn, Fisch, Geflügel, Nüsse).
Die Patienten wurden in vier Kategorien erfasst: Solche mit systolischen Blutdruck-Ausgangswerten $<130 \mathrm{mmHg}$, $130-139 \mathrm{mmHg}, 140-149 \mathrm{mmHg}$ und $>$ $150 \mathrm{mmHg}$.

In allen Interventionsgruppen sank der Blutdruck jeweils in allen vier Gruppen, berichtete Studienautor Stephen Juraschek:

- Bei Salzreduktion um 3,2 mmHg, $8,6 \mathrm{mmHg}, 9,0 \mathrm{mmHg}$ und $7 \mathrm{mmHg}$.

- Bei DASH-Diät um 4,5 mmHg, 4,3 mmHg, 4,7 $\mathrm{mmHg}$ und 10,6 $\mathrm{mmHg}$.
- Bei Salzreduktion plus DASH-

Diät um 5,3 mmHg, 7,5 mmHg,

$9,7 \mathrm{mmHg}$ und $20,8 \mathrm{mmHg}$.

Gerade bei Blutdruckwerten, die nach aktuellen europäischen und deutschen Leitlinien einer Hypertonie entsprechen (> $140 \mathrm{mmHg}$ ), waren die Effekte erheblich. Die Ergebnisse sollten nach Ansicht der Autoren Motivation sein, bei Hypertonie nicht müde zu werden, den Patienten von der Notwendigkeit einer Ernährungsumstellung zu überzeugen. - DE

- American Heart Association, Scientific Sessions 2017

\section{Prophylaxe-Maßnahmen empfohlen}

\section{Langes Fernsehen prädestiniert für tiefe Venenthrombosen}

\section{Fernsehen ist offenbar ein nicht un- wesentlicher Risikofaktor für tiefe Venenthrombosen.}

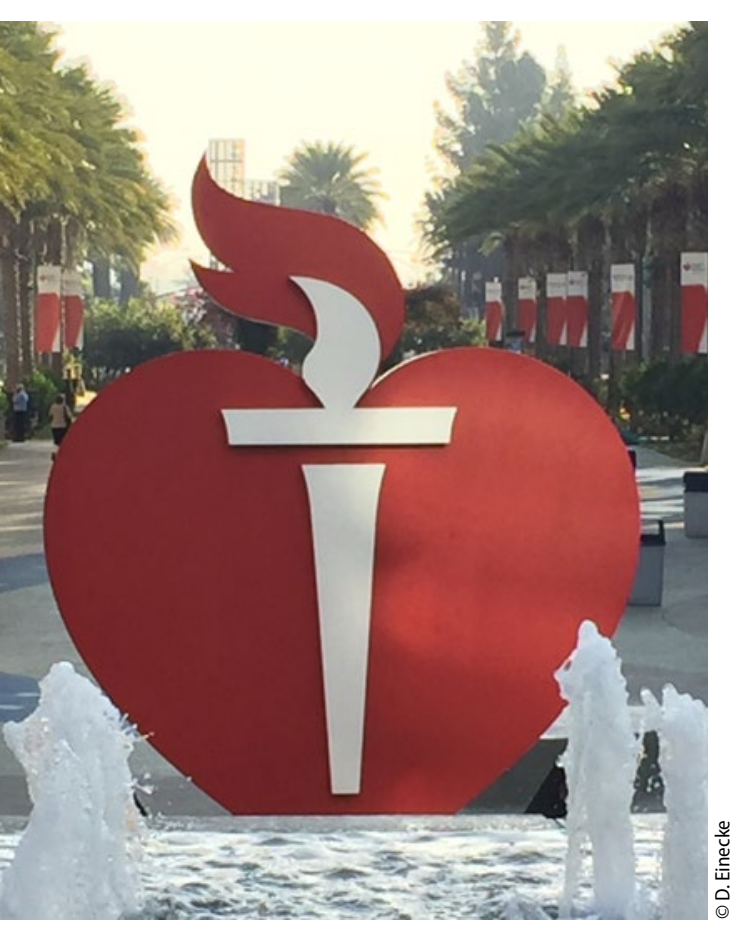

Wie Forscher von der University of Vermont in Burlington berichteten, bleibt das Thrombose-Risiko von Leuten, die viel Zeit vor dem Fernseher verbringen, auch dann erhöht, wenn sie täglich das von Leitlinien empfohlene Quantum körperliche Bewegung absolvieren.

„Fernsehen an sich mag nicht schädlich sein, aber wir neigen dazu, dabei sehr lange still zu sitzen und Snacks zu essen“, so die Studienautorin Mary Cushman.

Regelmäßiges langes Fernsehen ist früheren Studien zufolge bereits mit einem erhöhten Risiko für Herzkrankheiten assoziiert. Nun kommt noch ein erhöhtes Thromboserisiko hinzu.

In der Studie war eine Kohorte von gut 15.000 Personen prospektiv über 300.000 Personen-Jahre beobachtet worden. In dieser Zeit erlitten 691 Patienten eine venöse Thromboembolie (VTE). Nach multivariater Adjustierung für andere potenzielle Risikofaktoren zeigte sich eine positive Dosis-Wirkungs-Be- ziehung zwischen der vor dem Fernseher verbrachten Zeit und Thrombosen. Wer viel fernsah, hatte ein um den Faktor 1,7 bis 1,8 erhöhtes Risiko gegenüber Personen, die wenig oder nie fernsahen. Der Effekt war teilweise durch Übergewicht vermittelt.

Die Autoren empfehlen allerlei Strategien zur Vorbeugung: Fernsehen einschränken, später am Tag mit dem Fernsehen beginnen (und Lieblingssendungen ggf. aufzeichnen), häufiger beim Fernsehen aufstehen und umherlaufen bzw. sich durchbewegen, den Hometrainer vor den Fernseher stellen, körperliche Aktivität insgesamt erhöhen und Gewicht abnehmen.

Bei Personen mit erhöhtem Risiko für VTEs, etwa nach Operationen, Geburten, stattgehabten Thrombosen oder mit Krebserkrankung, empfehlen sie zusätzlich Thrombosestrümpfe und/oder Antikoagulation, sofern eine Neigung zu langen TV-Zeiten besteht. 